\title{
Is Pes Cavus Alignment Associated With Lisfranc Injuries of the Foot?
}

\author{
Jeremy D. Podolnick MD, Daniel S. Donovan MD, Nicholas DeBellis MD, \\ Alejandro Pino MD
}

Received: 20 May 2016/Accepted: 13 October 2016/Published online: 28 October 2016

(C) The Association of Bone and Joint Surgeons (B) 2016

\begin{abstract}
Background Lisfranc (tarsometatarsal joint) injuries are relatively rare, accounting for less than $1 \%$ of all fractures, and as many as $20 \%$ of subtle Lisfranc injuries are missed at the initial patient presentation. An undiagnosed Lisfranc injury can have devastating consequences to the patient. Therefore, any factor that can raise a clinician's index of suspicion to make this diagnosis is potentially important. The cavus foot has been associated with various maladies of the lower extremity, but to our knowledge, it has not been reported to be associated with Lisfranc injury.

Questions/purposes Do patients who experience a lowenergy Lisfranc injury have greater talar head coverage and a greater talo-first metatarsal angle than control subjects?
\end{abstract}

Each author certifies that he or she, or a member of his or her immediate family, has no funding or commercial associations (eg, consultancies, stock ownership, equity interest, patent/licensing arrangements, etc) that might pose a conflict of interest in connection with the submitted article.

All ICMJE Conflict of Interest Forms for authors and Clinical Orthopaedics and Related Research ${ }^{\circledR}$ editors and board members are on file with the publication and can be viewed on request.

Each author certifies that his or her institution approved or waived approval for the human protocol for this investigation and that all investigations were conducted in conformity with ethical principles of research

This study was performed at Mount Sinai St. Luke's-Mount Sinai West Hospital Center, New York, NY, USA.

J. D. Podolnick ( $₫)$, D. S. Donovan, N. DeBellis

Department of Orthopaedic Surgery, Mount Sinai St. Luke'sMount Sinai West, 1000 10th Avenue, New York, NY 10019, USA

e-mail: Jeremy.podolnick@gmail.com

A. Pino

Miami Institute for Joint Reconstruction, Miami, FL, USA
Methods A retrospective, case-control study was conducted from September 2011 to December 2014 to identify patients diagnosed and treated for a low-energy Lisfranc injury. Twenty-three adult patients with an average age of 42.6 years (SD, 16.3 years) were identified and compared with 61 adult control subjects with an average age of 49.4 years (SD, 14.1 years). Control subjects came from the practice of a fellowship-trained foot and ankle orthopaedic surgeon. Control subjects underwent a history and physical, clinical examination, and diagnostic imaging to confirm that they had no prior foot disorder, no prior foot surgeries, were within 3 years of age of a patient with a Lisfranc injury, and were independent ambulators. Two authors (DSD and JDP) measured the talonavicular and talo-first metatarsal angles on weightbearing AP and lateral radiographs of the foot. The intrarater reliability and interrater reliability for the talo-first metatarsal angle and the talonavicular angle showed high agreement. The intrarater intraclass correlation coefficients (ICC) of the talo-first metatarsal angle were 0.94 (95\% CI, 0.91-0.96) and 0.93 (95\% CI, 0.9-0.96). For the talonavicular angle the ICCs were 0.83 (95\% CI, 0.75-0.89) and 0.88 (95\% CI, 0.81$0.92)$ for Raters 1 and 2 respectively. The interrater ICCs were 0.91 (95\% CI, 0.69-0.96) for the talo-first metatarsal angle and 0.9 (95\% CI, 0.85-0.94) for the talonavicular angle. The patients and controls were compared to determine if the patients who sustained a Lisfranc injury were more likely to have a pes cavus foot alignment. We performed a mixed modeling analysis to control for potential cofounding variables and determine if there was an association of Lisfranc injury with the talo-first metatarsal angle and the talonavicular angle.

Results After controlling for confounding variables such as the effect of the measurement round effect and the effect of the rater, our repeated measures analysis via mixed 
model showed patients were associated with a higher talofirst metatarsal angle than control subjects (adjusted least square mean for patients $=3.05$; for controls $=-2.65$; mean difference, 5.7; $\mathrm{p}=0.001)$. Repeated measures analysis via mixed model showed that patients also were associated with a more positive talonavicular angle than control subjects (adjusted least square mean for patients $=$ -4.83 , for controls $=-11$; mean difference, $6.17 ; \mathrm{p}=$ 0.002). Patients with Lisfranc injuries had a higher mean talo-first metatarsal angle than did control subjects $\left(1.9^{\circ} \pm\right.$ $7.9^{\circ}$ versus $-2.2^{\circ} \pm 7.3^{\circ}$; mean difference, $4.1^{\circ} ; 95 \% \mathrm{CI}$, $-7.7^{\circ}$ to $-0.5^{\circ} ; \mathrm{p}=0.028$ ), and less talar uncovering $\left(-4.2^{\circ} \pm 9.7^{\circ}\right.$ versus $-11^{\circ} \pm 8^{\circ}$; mean difference, $6.7^{\circ}$; $95 \% \mathrm{CI},-6.7^{\circ}$ to $\left.-10.8^{\circ} ; \mathrm{p}=0.001\right)$.

Conclusions We found that cavus midfoot alignment was more prevalent among patients with Lisfranc injuries than among individuals with no foot injury or disorder. Although this does not suggest that cavus alignment causes or predisposes patients to this injury, we believe the finding is important because this provides a radiographic parameter that clinicians can use to raise their index of suspicion for a Lisfranc injury and aggressively pursue this diagnosis. Future studies would benefit from obtaining contralateral foot imaging at the time of injury in all patients with Lisfranc injury or prospectively following patients with foot imaging and recording the incidence of future foot injury.

Level of Evidence Level III, prognostic study.

\section{Introduction}

The Lisfranc joint complex, which is the articulation of the metatarsals to the cuneiforms and cuboid, joins the forefoot and the midfoot and is composed of bony and ligamentous elements which provide stability. The architecture of the complex is a key element in rendering stability to the foot $[8,13]$. Lisfranc (tarsometatarsal joint) injuries are relatively rare, accounting for less than $1 \%$ of fractures, and have been reported at a rate of one per 55,000 fractures [5]. Up to $20 \%$ of subtle Lisfranc injuries are missed on initial presentation [4, 15]. Lisfranc injuries may be caused by direct and indirect mechanisms of injury [17]. Indirect traumatic Lisfranc injuries typically occur after low-energy falls or twisting injury, whereas high-energy falls from height, direct crush, or motor vehicle accidents may lead to a more direct mechanism of injury [16]. Failure to achieve and maintain an anatomic reduction may lead to pain and disability $[4,5,18]$. Even subtle or undetectable displacement on radiographs may result in articular and physiologic changes [7].

Given the rarity of the injury, efforts have been made to identify predisposing anatomic factors, which may aid healthcare providers in pursuing a thorough workup to rule out this injury. To our knowledge, the relationship between cavus alignment and Lisfranc injury has not been reported. We thought it worth exploring because cavus feet have been associated with various foot and ankle disorders such as plantar fasciitis, medial longitudinal arch pain, iliotibial band syndrome, stress fractures, metatarsalgia $[2,6]$, recurrent ankle sprains with lateral instability, and lateral foot overloading [12]. Additionally, the biomechanics of a cavus foot increase the stress experienced at the tarsometatarsal joint complex. Characteristic findings of a cavus foot included varus of the hindfoot, plantar flexion of the first ray, and adduction and varus of the forefoot. The combination of these findings leads to a rigid foot that may be unable to accommodate abnormal stresses throughout the gait cycle [2,9]. Radiographic hallmarks of a cavus foot include a talo-first metatarsal angle greater than $5^{\circ}$ $[2,14]$, increased forefoot abduction as represented by the talonavicular angle [19], calcaneal pitch greater than $30^{\circ}$ $[1,2,14]$, talocalcaneal angle less than $20^{\circ}[14]$, and a posterior position of the fibula $[1,2]$. Previously established predisposing anatomic factors to Lisfranc injury include ratio of the second metatarsal length to overall foot length [3] and depth of the mortise of the second metatarsal between the lateral and medial cuneiforms [11].

We therefore sought to determine whether patients who experience a low-energy Lisfranc injury are more likely to have cavus foot alignment, that is, to have greater talar head coverage and a greater talo-first metatarsal angle than control subjects.

\section{Patients and Methods}

A retrospective, case-control study of patients with a Lisfranc injury was conducted from September 1, 2011 to December 1, 2014. All patients were evaluated by one foot and ankle fellowship-trained orthopaedic surgeon (AP). Diagnosis of a Lisfranc injury was made clinically and with radiographs, CT, and/or MRI, if indicated, and confirmed with the finding of intraoperative instability in patients treated operatively at our institution.

Lisfranc injuries diagnosed during the study period by the senior author (AP) and had appropriate imaging were included for review. Patients were identified based on a chief complaint of foot pain or swelling and who had weightbearing radiographs or advanced imaging confirming the diagnosis of a Lisfranc injury. Patients were excluded from analysis if they had no available weightbearing radiographs, sustained high-energy injury, had a diagnosis of neuromuscular pes cavus, were younger than 18 years, or had prior foot reconstructive surgery. Based on exclusion criteria, three patients were excluded because 
Table 1. Comparison of proportions of each variable (age, gender, laterality) by group (Lisfranc or control)

\begin{tabular}{llll}
\hline Variable & Lisfranc $(\mathrm{n}=23)$ & Control $(\mathrm{n}=61)$ & $\mathrm{p}$ Value \\
\hline Age* (year) & $42.6 \pm 16.3$ & $49.4 \pm 14.1$ & 0.299 \\
Gender & $15(65.2 \%)$ & $30(49.2 \%)$ & 0.189 \\
Male & $8(34.8 \%)$ & $31(50.8 \%)$ & \\
Female & & $34(55.7 \%)$ & 0.949 \\
Laterality & $13(56.5 \%)$ & $27(44.3 \%)$ & \\
Right & $10(43.5 \%)$ & \\
Left & & \\
\hline
\end{tabular}

* Presented as mean and SD.

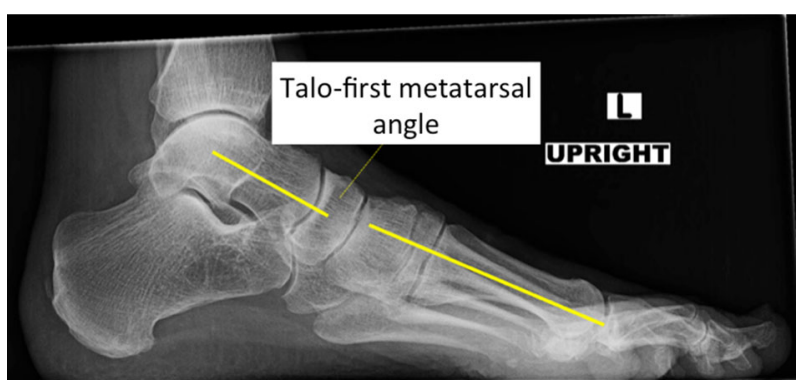

Fig. 1 This image shows measurement of the talo-first metatarsal angle on a weightbearing lateral foot radiograph.

they did not have pre- or postoperative weightbearing imaging. The mean age of the patients with Lisfranc injury was 42.6 years (SD, 16.3 years). There were eight females and 15 males in the Lisfranc group. All patients were able to independently ambulate preinjury. Initial postoperative $\mathrm{AP}$ and lateral weightbearing radiographs of the foot were used for analysis of patients with Lisfranc injury who did not have preoperative weightbearing imaging.

Control subjects were patients seen in the clinic of the senior author during the study period. A query of the senior author's patient database was performed to identify all patients with a diagnosis of Achilles tendinitis to avoid including patients with a disorder that may predispose them to a certain foot alignment. All control subjects presented initially for foot and/or ankle pain and had undergone a history and physical examination and imaging as dictated. Control subjects were excluded if they did not have weightbearing AP and lateral radiographs of the foot, had a prior diagnosis of a foot disorder, had prior foot surgery, or were younger than 18 years. Control subjects also were excluded if they were not within 3 years of age of a patient with a Lisfranc injury and if they were not independent ambulators. Our goal was to match control subjects with patients with a Lisfranc injury three-to-one based on age and gender. After applying our inclusion and exclusion criteria, there was a total of 61 control subjects for review. The mean age of the control subjects was 49.4 years (SD,

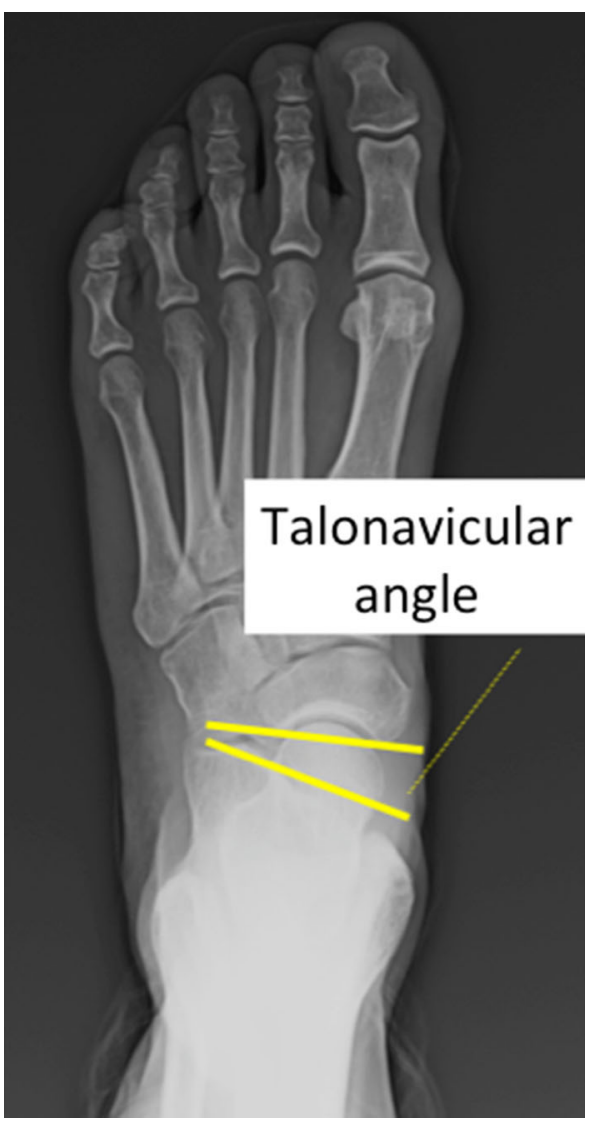

Fig. 2 Measurement of the talonavicular angle on a weightbearing AP foot radiograph is shown in this image.

14.1 years), and there were 31 females and 30 males in the control group.

The percentages of patients in the Lisfranc and control groups were analyzed based on age, gender, and laterality. We did not detect any associations between demographics and case-control status (Table 1).

All radiographs were available on our institution's picture archiving communication system (PACS). Two measurements were performed on weightbearing radiographs of the foot using the PACS integrated digital angle-measuring tool. 
The talo-first metatarsal angle (Meary's angle) was measured on the lateral radiograph. This was measured as the angle formed by a line originating from the center of the body of the talus, bisecting the talar neck and head, and the line through the longitudinal axis of the first metatarsal (Fig. 1). The talonavicular angle was measured on the AP radiograph of the foot. This was defined as the angle created by a line

Table 2. Intraobserver reliability

\begin{tabular}{lll}
\hline Variable & ICC & $95 \%$ CI \\
\hline Rater 1 & & \\
$\quad$ Talo-first metararsal angle & 0.94 & $0.91-0.96$ \\
$\quad$ Talonavicular angle & 0.83 & $0.75-0.89$ \\
Rater 2 & & \\
Talo-first metatarsal angle & 0.93 & $0.9-0.96$ \\
Talonavicular angle & 0.88 & $0.81-0.92$ \\
\hline
\end{tabular}

ICC = intraclass correlation coefficient.

Table 3. Interobserver reliability

\begin{tabular}{lll}
\hline Variable & ICC & $95 \%$ CI \\
\hline Talo-first metatarsal angle & 0.91 & $0.69-0.96$ \\
Talonavicular angle & 0.9 & $0.85-0.94$ \\
\hline
\end{tabular}

ICC = intraclass correlation coefficient. connecting the most medial and most lateral aspect of the articular surface of the navicular and a line connecting the most medial and most lateral articular surface of the talar head at the talonavicular joint (Fig. 2). Uncoverage of the talus was measured as a negative number.

Two authors (DSD and JDP), both postgraduate-year IV residents, performed all measurements. Overall, two rounds of measurements were performed for patients and control subjects. Measurement rounds were separated by a period of 2 weeks. All measurements in each round were performed in one session to control for potential measurement error and bias. The authors were blinded to the coauthor's measurements and their own prior measurements. The same radiograph was measured in both sessions.

The intraclass correlation coefficient (ICC) was used to describe the interrater and intrarater reliability for measurement of the talo-first metatarsal angle and talonavicular angle. On the ICC analysis of intrarater reliability, the talofirst metatarsal measurements for Rater 1 had an ICC of 0.94 (95\% CI, 0.91-0.96) and talonavicular measurements had an ICC of 0.83 (95\% CI, 0.75-0.89), reflecting high intrarater reliability. The talo-first metatarsal measurements for Rater 2 had an ICC of 0.93 (95\% CI, 0.90-0.96) and talonavicular measurements had an ICC of 0.88 (95\% CI, 0.81-0.92), reflecting high intrarater reliability (Table 2). Interrater reliability of the talo-first metatarsal angle was 0.91 (95\% CI, 0.69-0.96) and of the talonavicular angle

Table 4. Comparison of the talo-first metatarsal angle and the talonavicular angle for patients and control subjects

\begin{tabular}{|c|c|c|c|c|c|c|c|c|}
\hline \multirow[t]{2}{*}{ Rater } & \multirow[t]{2}{*}{ Variable } & \multicolumn{2}{|l|}{ Patient } & \multicolumn{2}{|l|}{ Control } & \multicolumn{2}{|c|}{ Mean difference } & \multirow[t]{2}{*}{ T-test } \\
\hline & & Mean & SD & Mean & SD & Mean & $95 \%$ CI & \\
\hline \multirow[t]{8}{*}{1} & Talo-first metatarsal angle & & & & & & & \\
\hline & Round 1 & 3.9 & 7.7 & -1.2 & 7 & -5 & -8.5 to -1.5 & 0.0052 \\
\hline & Round 2 & 2.9 & 7.5 & -1.6 & 7.1 & -4.5 & -8 to -1 & 0.0123 \\
\hline & Average & 3.4 & 7.5 & -1.35 & 6.9 & -4.8 & -8.2 to -1.3 & 0.0072 \\
\hline & Talonavicular angle & & & & & & & \\
\hline & Round 1 & -2.5 & 9.8 & -10.8 & 9.1 & -8.3 & -8.3 to -12.8 & 0.0004 \\
\hline & Round 2 & -5.3 & 11.3 & -11.3 & 7.7 & -6.1 & -6.1 to -10.4 & 0.006 \\
\hline & Average & -3.9 & 10.2 & -11.1 & 8 & -7.2 & -7.2 to -11.4 & 0.001 \\
\hline \multirow[t]{8}{*}{2} & Talo-first metatarsal angle & & & & & & & \\
\hline & Round 1 & 1.1 & 9.2 & -3 & 7.9 & -4.1 & -4.1 to -8.2 & 0.0462 \\
\hline & Round 2 & -0.4 & 8.4 & -3.1 & 8.1 & -2.7 & -2.7 to -6.7 & 0.1782 \\
\hline & Average & 0.3 & 8.6 & -3.1 & 7.9 & -3.4 & -3.4 to -7.4 & 0.0887 \\
\hline & Talonavicular angle & & & & & & & \\
\hline & Round 1 & -4.4 & 8.8 & -10.5 & 8.3 & -6.2 & -6.2 to -10.3 & 0.0037 \\
\hline & Round 2 & -4.8 & 11.4 & -11.1 & 9.1 & -6.3 & -6.3 to -11.1 & 0.0098 \\
\hline & Average & -4.6 & 9.7 & -10.8 & 8.4 & -6.2 & -6.2 to -10.5 & 0.0048 \\
\hline \multirow[t]{2}{*}{ Average } & Talo-first metatarsal angle & 1.9 & 7.9 & -2.2 & 7.3 & -4.1 & -7.7 to -0.5 & 0.0283 \\
\hline & Talonavicular angle & -4.2 & 9.7 & -11 & 8 & -6.7 & -6.7 to -10.8 & 0.0017 \\
\hline
\end{tabular}

Mean, SD, and CI presented in degrees. 
was 0.9 (95\% CI, 0.85-0.94), reflecting high agreement (Table 3).

The four talo-first metatarsal angle measurements were averaged and examined to define the patients in each group with pes cavus. Pes cavus was defined by a talo-first metatarsal angle greater than $5^{\circ}$. Pes cavus was more common in the patients compared with the control subjects. Seven of 23 patients had a talo-first metatarsal angle greater than $5^{\circ}(30.4 \%)$ and only seven of 61 control subjects had a talo-first metatarsal angle greater than $5^{\circ}$ (11.5\%) (0.050).

A T-test was used to compare angular measurements between rater per round and the averaged measurements calculated by the raters. Angle measurements were averaged for each patient. The T-test was used to compare the average talo-first metatarsal angle and talonavicular angle of patients with Lisfranc injuries with those of control subjects. Patients with Lisfranc injuries had a higher mean talo-first metatarsal angle than did control subjects $\left(1.9^{\circ} \pm\right.$ $7.9^{\circ}$ versus $-2.2^{\circ} \pm 7.3^{\circ}$; mean difference, $4.1^{\circ}$; $95 \% \mathrm{CI}$, $-7.7^{\circ}$ to $-0.5^{\circ} ; \mathrm{p}=0.028$ ). Analysis of the mean talonavicular angle showed that patients with Lisfranc injuries had less talar uncovering than control subjects $\left(-4.2^{\circ} \pm\right.$ $9.7^{\circ}$ versus $-11^{\circ} \pm 8$; mean difference, $6.7^{\circ} ; 95 \% \mathrm{CI}$, $-6.7^{\circ}$ to $-10.8^{\circ} ; \mathrm{p}=0.001$ ) (Table 4 ). In each round of measurements, the mean talo-first metatarsal angle of the Lisfranc group was higher than that of the control group and the Lisfranc group showed less talar uncovering than the control group (Table 4).

We analyzed the talo-first metatarsal angle and talonavicular angle by round and rater to determine if difference existed by case-control status. To examine the overall relationship between angle and case-control status, adjusting for round and rater, we performed repeated measures analysis via a mixed model to generate and compare adjusted least square means for the talo-first metatarsal angle (Fig. 3) and talonavicular angle (Fig. 4).

\section{Results}

Each angular measurement was performed twice by two raters, giving a total of four measurements for each talofirst metatarsal angle and talonavicular angle. The mixed modeling analysis allowed us to control for the repeated nature of the data. After controlling for the effect of the measurement round and the effect of the rater on the talofirst metatarsal and the talonavicular angular measurements via a repeated measures analysis, we found that Lisfranc injury was strongly associated with a higher talo-first metatarsal angle in patients compared with the angle for control subjects (adjusted least square mean for patients = 3.05; for control subjects $=-2.65$; mean difference, $5.7 ; \mathrm{p}$

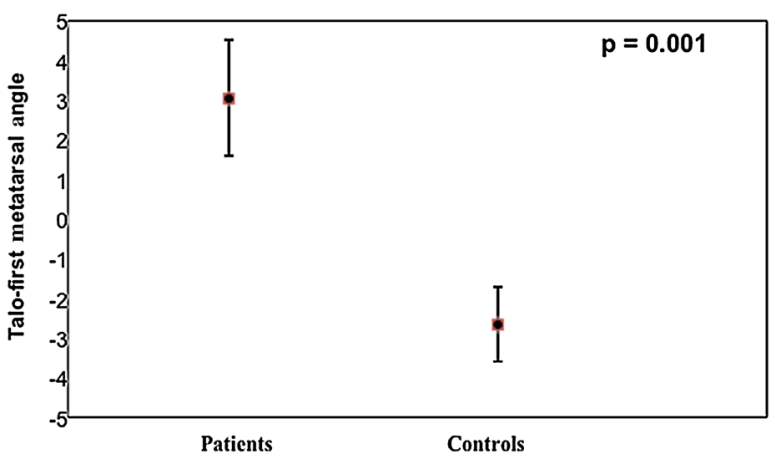

Fig. 3 Least square means of the talo-first metatarsal angle in patients and control subjects, adjusted for rater and round are shown. The bars show standard error. $\mathrm{P}$ is the $\mathrm{p}$ value for the patient-control difference.

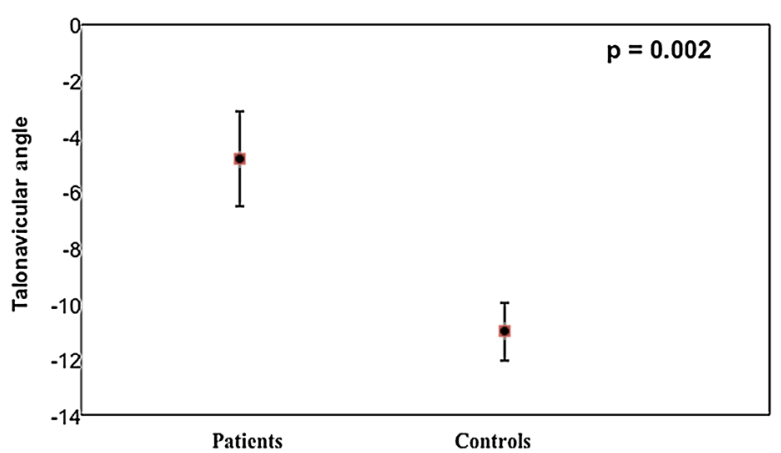

Fig. 4 Least square means of the talonavicular angle in patients and control subjects, adjusted for rater and round, are shown. The bars show standard error. $\mathrm{P}$ is the $\mathrm{p}$ value for the patient-control difference.

$=0.001$ ) (Fig. 3). Based on the same mixed modeling analysis, we found that the difference between the talo-first metatarsal angles of patients and control subjects was associated with the rater (adjusted least square mean for Rater $1=1.23$; for Rater $2=-0.84$; mean difference, $2.1 ; \mathrm{p}$ $<0.001$ ) and with the measurement round (adjusted least square mean for round $1=0.45$; for round $2=-0.05$; mean difference, $0.51 ; \mathrm{p}=0.254$ ). In other words, the talo-first metatarsal angle was measured to be higher by Rater 1 compared with Rater 2, and the talo-first metatarsal angle was measured to be higher in round 1 compared with round 2. This would suggest that the observed differences are driven in part by actual differences between patients with injuries and control subjects, and also by variability in measurements performed during successive rounds of measurements, and by rater bias. However, our previously reported ICC of intrarater reliability for the talo-first metatarsal angle was 0.94 (95\% CI, 0.91-0.96) and our ICC of interrater reliability for the talo-first metatarsal angle was 0.91 (95\% CI, 0.69-0.96), both of which reflect high agreement. Our ICC analysis of intrarater and interrater reliability would support the assertion that there was 
no association of the talo-first metatarsal angle with rater or round of measurement.

Our repeated measures analysis showed that patients also were associated with a more positive talonavicular angle than control subjects (adjusted least square mean for patients $=-4.83$; for controls $=-11$; mean difference, $6.17 ; \mathrm{p}=$ 0.002) (Fig. 4). We did not find an association of the talonavicular angle with raters (adjusted least square mean for rater $1=-7.9$; for rater $2=-7.94$; mean difference, $0.04 ; \mathrm{p}=$ 0.935 ) or an association with the measurement round (adjusted least square mean for round $1=-7.52$; for round $2=$ -8.31 ; mean difference, $0.79 ; \mathrm{p}=0.077$ ).

\section{Discussion}

Tarsometatarsal injuries can occur by high- or low-energy mechanisms. Anatomic characteristics may play a role in the likelihood of low-energy mechanisms resulting in a Lisfranc injury. Although many pathologic conditions have been associated with pes cavus including stress fractures of the metatarsals, vertical fracture of the medial malleolus, inversion sprains of the ankle, peroneal tendon disorders, lateral overload, and hallux sesamoiditis [2, 6, 12], there has been no previously noted relationship between pes cavus and Lisfranc injury. We wondered whether the rigid nature of a locked hindfoot seen in cavus feet does not allow for dissipation of stresses seen with low-energy injury [1], which subsequently might be passed to the Lisfranc joint complex. We therefore sought to determine whether cavus alignment was more common among patients with Lisfranc injuries than in uninjured control subjects. We found that a greater percentage of patients in the Lisfranc group had a talo-first metatarsal angle greater than $5^{\circ}$, meeting a definition of pes cavus. Overall, patients in the Lisfranc group had higher talofirst metatarsal angles and less talar uncoverage. Based on these findings, we believe a pes cavus foot alignment is associated with Lisfranc injury.

The two main weaknesses of this study were that its retrospective design only allowed us to infer association rather than causation and that the control group was not well matched. The retrospective analysis of this group of patients allowed for potential biases to influence the study outcome. The Lisfranc injury group was limited to patients treated and diagnosed by the senior author (AP). Although unlikely, it is possible that more subtle injuries were missed. Based on our conclusion that the patients with injury tended to have more cavus feet, it is possible that we have underreported this association. Additionally, the raters (DSD and JDP) were not blinded to the patients in the injury and control groups because we were forced to use postoperative radiographs for the patients with Lisfranc injury. However, the surgeon's operative protocol is to perform a tarsometatarsal arthrodesis restoring the patient's native anatomic position. Additionally, the talonavicular angle should not be affected by surgical fixation and therefore would reflect the preinjury alignment of the foot. Because we used postoperative radiographs instead of the contralateral limb, we were unable to perform a blinded study. However, each rater performed the talo-first metatarsal and talonavicular measurements twice, 2 weeks apart. We also blinded the raters to the other's measurements and their prior measurements in an attempt to control for potential bias. A future study would benefit from obtaining radiographs of the contralateral, uninjured limb in patients with Lisfranc injury. Additionally, we had some limitations when matching the control group with the patients. We attempted to match control subjects with patients by age and gender; however, after applying our inclusion and exclusion criteria to the patients seen in the attending's clinic, we had a limited population from which to select. Control subjects tended to be older and there was a higher percentage of males in the control group. However, no associations between demographics and casecontrol status were found (Table 1). All control subjects were independent ambulators and were within 3 years of age of one of the patients with Lisfranc injury. No control subject had a prior diagnosis of a foot disorder or prior foot surgery, and subjects in the control population were included only if they had a diagnosis of a disorder proximal to the foot (ankle sprain or Achilles tendinitis). Additionally, to our knowledge, there has not been a proven association between pes cavus and age or gender.

Investigations of other anatomic predisposing factors to Lisfranc injury have included second metatarsal length and depth of the mortise of the second metatarsal between the medial and middle cuneiforms. Gallagher et al. [3] compared 26 patients with known ligamentous Lisfranc injuries with 52 control subjects. An association was found between Lisfranc injury and a smaller ratio of second metatarsal length to overall foot length. They hypothesized that a decrease in this ratio increased torsional stresses at the Lisfranc joint. The difference in calcaneal pitch between control subjects and patients with Lisfranc injury approached significance $(\mathrm{p}=0.08)$, which may have been related to patient sample size; however, the difference between first metatarsal to talus angle was equivalent ( $\mathrm{p}=$ 0.50). The talus to first metatarsal angle reflects forefoot abduction, which is similar to our measure of the talonavicular angle. Gallagher et al. [3] did not find an association of the talus to first metatarsal angle to Lisfranc injury, which is in contrast to our finding. Peicha et al. [11] investigated 33 patients with injuries to the tarsometatarsal joint and 84 cadaver specimens as a control. The injury group was found to have a decreased depth of the mortise of the Lisfranc joint between the lateral and medial 
cuneiforms. We found that a cavus foot alignment was associated with Lisfranc injury. To our knowledge, this is a novel finding and the association between Lisfranc injuries and cavus feet has not previously been reported.

In a cavus foot, the foot remains locked with the hindfoot in inversion throughout the gait cycle. Thus, the normal ability of the foot to evert at the subtalar joint and for the midfoot to pronate is lost. The transverse tarsal joints remain locked, leading to a stiff midfoot. The combination of these altered biomechanics causes inability of normal stress dissipation at the transverse tarsal joint and leads to increased stress at the Lisfranc joint [2,9]. In patients with cavus feet, we theorize that the Lisfranc joint experiences higher than normal stresses throughout the gait cycle and therefore may be more susceptible to injury. However, our study did not prove that this was the case; our study design could not evaluate cause and effect. Future studies might do this by performing large-scale prospective studies to evaluate the incidence of Lisfranc injury and foot alignment.

Untreated, Lisfranc injuries can lead to arthritis, instability, and chronic pain [14, 15]. Even subtle or undetectable displacement on radiographs may result in articular and physiologic changes [7]. Given the rarity of the injury, efforts have been made to identify predisposing anatomic factors, which may aid healthcare providers in pursuing a thorough workup to rule out this injury. Peicha et al. [11] concluded that the shallower the mortise of the Lisfranc joint, the greater risk of Lisfranc injury. Gallagher et al. [3] reported that occurrence of a ligamentous Lisfranc injury was associated with a smaller second metatarsal length to foot length. Our study provides an additional radiographic parameter that clinicians can use to raise their index of suspicion for Lisfranc injury. Patients who present with equivocal midfoot findings and a cavus foot should be liberally referred for advanced imaging. Additionally, Gallagher et al. [3] suggested that this knowledge of second metatarsal to foot length ratio could be used to direct susceptible individuals away from certain activities. They suggested that populations at a higher incidence of injury, such as football players who sustain Lisfranc injuries at a rate of $4 \%$ per year [10], might benefit from a screening examination of the foot. Clinicians should be aware that when treating patients involved in these activities, a cavus foot should prompt aggressive investigation of midfoot complaints after injury.

The results of our study show that patients who sustain a low-energy Lisfranc injury are more likely than control subjects to have a talo-first metatarsal angle greater than $5^{\circ}$, and when compared with control subjects, have greater talofirst metatarsal angles. Patients with a Lisfranc injury are also more likely to have a greater amount of coverage of the talar head compared with uninjured patients, reflecting a cavus foot alignment. Clinicians should have a higher index of suspicion of a Lisfranc injury when evaluating patients with cavus feet after low-energy injury and midfoot symptoms.

\section{References}

1. Abbasian A, Pomeroy G. The idiopathic cavus foot: not so subtle after all. Foot Ankle Clin. 2013;18:629-642.

2. Aminian A, Sangeorzan BJ. The anatomy of cavus foot deformity. Foot Ankle Clin. 2008;13:191-198.

3. Gallagher SM, Rodriguez NA, Anderson CR, Granberry WM, Panchbhavi VK. Anatomic predisposition to ligamentous Lisfranc injury: a matched case-control study. J Bone Joint Surg Am. 2013;95:2043-2047.

4. Goossens M, De Stoop N. Lisfranc's fracture-dislocations: etiology, radiology, and results of treatment. A review of 20 cases. Clin Orthop Relat Res. 1983;176:154-162.

5. Hardcastle PH, Reschauer R, Kutscha-Lissberg E, Schoffmann $\mathrm{W}$. Injuries to the tarsometatarsal joint: incidence, classification and treatment. J Bone Joint Surg Br. 1982;64:349-356.

6. Ledoux WR, Shofer JB, Ahroni JH, Smith DG, Sangeorzan BJ, Boyko EJ. Biomechanical differences among pes cavus, neutrally aligned, and pes planus feet in subjects with diabetes. Foot Ankle Int. 2003;24:845-850.

7. Lu J, Ebraheim NA, Skie M, Porshinsky B, Yeasting RA. Radiographic and computed tomographic evaluation of Lisfranc dislocation: a cadaver study. Foot Ankle Int. 1997;18:351-355.

8. Mann R, Haskell A. Biomechanics of the foot and ankle. In: Couchlin M, Mann R, Saltzman C, eds. Surgery of the Foot and Ankle. Philadelphia, PA: Elsevier; 2007:3-44.

9. Manoli A 2nd, Graham B. The subtle cavus foot, "the underpronator". Foot Ankle Int. 2005;26:256-263.

10. Meyer SA, Callaghan JJ, Albright JP, Crowley ET, Powell JW. Midfoot sprains in collegiate football players. Am J Sports Med. 1994;22:392-401.

11. Peicha G, Labovitz J, Seibert FJ, Grechenig W, Weiglein A, Preidler KW, Quehenberger F. The anatomy of the joint as a risk factor for Lisfranc dislocation and fracture-dislocation: an anatomical and radiological case control study. J Bone Joint Surg Br. 2002;84:981-985.

12. Peters PG, Guyton GP. Metatarsal and calcaneal osteotomies for cavus foot. In: Kitaoka HB, ed. Master Techniques in Orthopaedic Surgery: The Foot and Ankle. Philadelphia, PA: Lippincott Williams \& Wilkins; 2013:301-314.

13. Richter M, Kwon JY, Digiovanni CW. Foot injuries. In: Browner BD, Jupiter JB, Krettek C, Anderson P, eds. Skeletal Trauma: Basic Science, Management, and Reconstruction. Philadelphia, PA: Elsevier; 2015:2251-2388.

14. Rosenbaum A, Dellenbaugh S, DiPreta J, Uhl R. Subtle injuries to the Lisfranc joint. Orthopedics. 2011;34:882-887.

15. Thompson MC, Mormino MA. Injury to the tarsometatarsal joint complex. J Am Acad Orthop Surg. 2003;11:260-267.

16. Vuori JP, Aro HT. Lisfranc joint injuries: trauma mechanisms and associated injuries. J Trauma. 1993;35:40-45.

17. Wiley JJ. The mechanism of tarso-metatarsal joint injuries. $J$ Bone Joint Surg Br. 1971;53:474-482.

18. Wilson DW. Injuries of the tarsometatarsal joints: etiology, classification and results of treatment. J Bone Joint Surg Br. 1972;54:677-686.

19. Witt BL, Bohay DR, Anderson JG. Lateral column lengthening. In: Wiesel SW, Parvizi J, Rothman RH, Easley ME, Hunt TR 3rd, eds. Operative Techniques in Orthopaedic Surgery. Philadelphia, PA: Lippincott Williams \& Wilkins; 2015:4505-4517. 